\title{
Morphologic and neurochemical alterations in the superior colliculus of the genetically epilepsy-prone hamster (GPG/Vall)
}

\author{
Verónica Fuentes-Santamaría ${ }^{a, *}$, Juan Carlos Alvarado ${ }^{a}$, \\ Antonio S. Herranz ${ }^{b}$, Natividad García-Atarés ${ }^{c}$, \\ Dolores E. López ${ }^{\mathrm{d}}$
}

\footnotetext{
a Department of Neurobiology and Anatomy, Wake Forest University School of Medicine, Winston-Salem, NC 27157-1010, USA b Servicio de Neurobiología, Departamento de Investigación, Hospital Ramon y Cajal, Ctra. Colmenar Viejo Km 9, 28034 Madrid, Spain

' Departamento de Anatomía y Radiología, Universidad de Valladolid, 47007 Valladolid, Spain

' Laboratorio de Neurobiología de la Audición, Departamento de Biología Celular y Patología, Instituto de Neurociencias de Castilla y León, Universidad de Salamanca, 37007 Salamanca, Spain
}

Received 25 March 2007; received in revised form 4 June 2007; accepted 7 June 2007

\section{KEYWORDS \\ Calcium-binding protein; \\ AGS; \\ Animal model; \\ Midbrain; \\ Densitometric \\ analysis}

\begin{abstract}
Summary The GPG/Vall hamster is an animal model that exhibits seizures in response to sound stimulation. Since the superior colliculus (SC) is implicated in the neuronal network of audiogenic seizures (AGS) in other forms of AGS, this study evaluated seizure-related anatomical or neurochemical abnormalities in the SC of the GPG/Vall hamster. This involved calbindin (CB) and parvalbumin (PV) immunohistochemistry, densitometric analysis and high performance liquid chromatography in the superficial and deep layers of the SC in control and epileptic animals. Compared to control animals, a reduction in SC volume and a hypertrophy of neurons located in the deep layers of the SC were observed in the epileptic hamster. Although, analysis of CB-immunohistochemistry in the superficial layers did not show differences between groups, analysis of PV-immunostaining in the deep SC revealed an increase in the mean gray level within immunostained neurons as well as a decreased immunostained neuropil in the GPG/Vall hamster as compared to control animals. These alterations were accompanied by a decrease in the levels of GABA and increased levels of taurine in the epileptic animal. These data indicate that the deep SC of the GPG/Vall hamster is structurally abnormal; suggesting its involvement in the neuronal network for AGS.
\end{abstract}

(C) 2007 Elsevier B.V. All rights reserved.

\footnotetext{
* Corresponding author. Tel.: +1 336713 0150; fax: +1 336 716-4534.

E-mail address: vfuentes@wfubmc.edu (V. Fuentes-Santamaría).
} 


\section{Introduction}

The genetically epilepsy-prone hamster (GPG/Vall) is an animal model that exhibits generalized tonic-clonic seizures in response to sound stimulation (Soria Milla et al., 1987; GilVerona et al., 1991). These seizures involve several stages including (1) a short latency period from the initiation of sound stimulation to the onset of an explosive burst of wild running, (2) a wild running phase where the hamster falls over on its side, (3) tonic-clonic convulsions and (4) postictal period (Macías Fernandez et al., 1992). Similar to other animal models of audiogenic seizures (AGS) (Penny et al., 1983, 1986; Roberts et al., 1985; Roberts and Ribak, 1986), several morphologic and neurochemical abnormalities have been observed in the auditory pathway of the GPG/Vall hamster (Fuentes-Santamaria et al., 2005a). These include atrophic changes starting in the cochlea and extending along the auditory brainstem as well as upregulation of calciumbuffering mechanisms in the inferior colliculus (IC) and in its afferent sources (Fuentes-Santamaria et al., 2005a).

During the last two decades, numerous pharmacological, lesion and electrophysiological studies have demonstrated that the IC is a critical structure implicated in audiogenic seizure susceptibility (Kesner, 1966; Wada et al., 1970; Browning, 1986; Faingold et al., 1986; Millan et al., 1986; Ribak et al., 1988). The central nucleus of the inferior colliculus (CNIC), in particular, has been proposed to be involved in AGS initiation (Willott and Lu, 1980) while the external cortex $(\mathrm{ECIC})$, which receives a dense projection from the ipsilateral CNIC (Coleman and Clerici, 1987), is implicated in the propagation of the seizures (Willott and Lu, 1980; Chakravarty and Faingold, 1996). In several genetic models of AGS, the ECIC mediates the seizure output pathway in the neuronal network for AGS (Le Gal La Salle and Naquet, 1990; McCown et al., 1984) through its connections with the superior colliculus (SC) (Van Buskirk, 1983; Garcia-Cairasco et al., 1993). The mammalian SC is a laminated, midbrain structure that can be functionally divided into superficial and deep laminae (for review, see Stein and Meredith, 1993). Multisensory neurons located in the deep layers, integrate information from different sensory modalities and send their descending axons to motor and premotor centers in the brainstem that control the position of the eyes, head and pinnae (Huerta and Harting, 1982; Moschovakis and Karabelas, 1985; Guitton and Munoz, 1991; Meredith et al., 2001; Fuentes-Santamaria et al., 2006). Because the activity of these multisensory neurons depends on the coordination of afferents from a number of morphologically diverse neuron types, including those from the inferior colliculus, it has been proposed that SC neurons may play a significant role in the efferent pathway implicated in the AGS (Faingold and Randall, 1999; Faingold and Casebeer, 1999).

Evidence suggests that the $\mathrm{SC}$ is a requisite nucleus for the propagation of AGS activity (Ross and Coleman, 2000). Supporting this idea, in situ hybridization studies to reveal cfos-mRNA have demonstrated increased labeling in the deep SC after sound-induced seizures in genetically epilepsyprone rats (GEPR-9s), suggesting the involvement of this structure in the progression of seizure activity (Ribak et al., 1997). Additional evidence for a critical role of the SC in AGS comes from pharmacological studies based on focal microin- jections of GABA antagonists into the deep SC of normal rats (Cools et al., 1984; Millan et al., 1986). These studies show that low dose injections of either picrotoxin or bicuculline can generate not only spontaneous seizure activity (Millan et al., 1986) but also wild running behavior (Cools et al., 1984). Interestingly, a rapid burst firing of neurons located in the deep layers of the SC has been observed in freely moving GEPRs with chronically implanted electrodes, suggesting that the deep layers play a key role in triggering the wild running behavior that begins AGS (Faingold and Randall, 1999). The specific role played by the SC in the neuronal network for AGS is partially unknown. Cellular factors including intracellular calcium concentration and inhibitory and excitatory neurotransmitters could also be affected in the SC of different animal models of AGS and thus, contributing to the generation of convulsive behaviors exhibited during seizures.

Accordingly, the present project investigates morphologic and neurochemical alterations in the SC of the GPG/Vall hamster by using immunocytochemical methods, densitometric analysis, and high performance liquid chromatography (HPLC). Specifically, our goal was to test the hypothesis that the deep layers of the SC, which contain multisensory neurons, present abnormalities that may have substantial effects in the efferent response required for the generation of motor seizures.

\section{Methods}

\section{Experimental animals}

Data were obtained from seven epileptic (GPG/Vall) and seven age-matched control hamsters (Golden Syrian hamster, Mesocricetus auratus). All animals (7-month-old males) were handled and cared for according to the Guidelines of the European Communities Council Directive (DOCE L222; 8-24-1999) and the current Spanish legislation for the use and care of laboratory animals (RD 233/88). Efforts were made to minimize the animals' discomfort and the number of animals used. The GPG/Vall hamster strain, which exhibits audiogenic seizures in response to sound stimulation, is housed at the University of Valladolid (Spain). Control hamsters were purchased from Charles River Company (France) 3 months before experiments were carried out to avoid bias in the analysis and interpretation of the distribution of immunostaining that could be due to either a new acoustic environment or to the effect of a stressful situation after shipment. All the animals used for immunocytochemistry and for HPLC were tested for audiogenic seizure susceptibility once every 15 days, for a total of 11 tests, under the same conditions. Because these seizures first appear around day 20 of postnatal life, the first seizure was induced at 30 days of age (Soria Milla et al., 1987). For seizure induction, the auditory stimulus was white noise with a frequency of $1-20 \mathrm{kHz}$, an intensity of $60-80 \mathrm{~dB}$ and duration of $20 \mathrm{~s}$ (Soria Milla et al., 1987). The last seizure was induced 1 month before they were sacrificed at 7 months of age. Control hamsters never exhibited sound-induced seizures. Hamsters were held under environmentally controlled conditions (06:00 a.m.:20:00 p.m. light/dark cycle, $25^{\circ} \mathrm{C}$, with food and water available ad libitum).

In order to determine morphologic and biochemical abnormalities in the SC of the GPG/Vall hamster compared to control animals, four sets of experiments were performed: (1) AChE and Nissl-staining were used to determine both laminar boundaries and possible atrophic changes in the SC, (2) calbindin and parvalbumin immunocytochemistry were carried out to study possible alterations in the distribution of these calcium-binding proteins in the superfi- 
cial layers and deep layers of the SC, respectively, (3) densitometry was used to quantify levels of calbindin and parvalbumin immunostaining within neurons and fibers and (4) HPLC was conducted to determine amino acid levels in this midbrain structure.

\section{Acetylcholinesterase (AchE) histochemistry}

Sections were rinsed with distilled water and incubated for $1 \mathrm{~h}$ in $50 \mathrm{ml}$ of a solution containing sodium acetate $(0.1 \mathrm{M}, \mathrm{pH} 5.0)$, copper sulfate, glycine, acetylthiocholine iodide and iso-OMPA. After incubation, sections were rinsed with distilled water, and placed for $2 \mathrm{~min}$ in a solution of $1.5 \%$ sodium sulfide. Then, sections were rinsed again and placed in $1 \%$ silver nitrate for $2 \mathrm{~min}$. After a final rinse, they were mounted, dried, cleared, and coverslipped.

\section{Anatomical boundaries}

In order to determine laminar boundaries within the SC, the last two series of sections were used for AChE as well as for Nisslstaining. One of the main advantages of AChE staining is that it predominantly stains fibers, which allows SC laminar boundaries to be easily determined (compare $1 \mathrm{~A}$ with $1 \mathrm{~B}$ ). Similar to other mammals (Kanaseki and Sprague, 1974), and in agreement with a previous study in the hamster (Chevalier and Mana, 2000), the SC is a laminated structure usually divided into superficial and deep layers. The superficial layers include the stratum zonale (SZ), stratum griseum superficiale (SGS), and stratum opticum (SO), while the deep layers consist of the stratum griseum intermediale $(\mathrm{SGI})$, the stratum album intermedium (SAI), the stratum griseum profundum (SGP) and the stratum album profundum (SAP).

\section{Immunocytochemistry}

Control $(n=3)$ and epileptic $(n=3)$ adult male golden hamsters were deeply anesthetized with pentobarbital $(60 \mathrm{mg} / \mathrm{kg})$ and perfused transcardially with a $0.9 \%$ saline wash followed by a fixative solution of $4 \%$ paraformaldehyde, $0.1 \%$ glutaraldehyde, and $15 \%$ saturated picric acid in $0.1 \mathrm{M}$ phosphate buffer $\mathrm{pH} 7.4(\mathrm{~PB})$. Brains were removed and cryoprotected for $48 \mathrm{~h}$ at $4{ }^{\circ} \mathrm{C}$ in $30 \%$ sucrose in PB. Coronal sections of $40 \mu \mathrm{m}$ thickness were sectioned on a freezing sliding microtome (Microm HM400; Leica, Heildelberg, Germany). SC sections from control and epileptic animals were collected in $\mathrm{PB}$ in four alternating series and processed simultaneously. The first two series of sections were incubated overnight at $4{ }^{\circ} \mathrm{C}$ in a primary antibody solution diluted in TBS (Tris-buffered saline, $\mathrm{pH} 7.6$ ) containing $0.2 \%$ Triton X-100 (TBS-Tx $0.2 \%$ ) to detect either calbindin (mouse anti-calbindin monoclonal antibody Sigma, St. Louis, MO, USA; $1: 1000$ ) or parvalbumin (mouse anti-parvalbumin monoclonal antibody Sigma, 1:1000). After four 15 min rinses in TBS-Tx $0.2 \% \mathrm{pH} 7.6$, sections were incubated in a solution of biotinylated secondary antibody $(1: 200)$ diluted in the same buffer (Vector Laboratories, Burlingame, CA, USA) for $2 \mathrm{~h}$ at room temperature. The Vector biotin-avidin procedure (ABC kit \#PK4000) was used to link the antigen-antibody complex to HRP, which was visualized by using the diaminobenzidine tetrahydrochloride (DAB, 0.01\%) peroxidase histochemistry procedure. Sections of control and epileptic hamsters were incubated 4-6 min in DAB-peroxidase solution in order to ensure an adequate interpretation of the results. Finally, the sections were mounted on gelatin-coated slides and dehydrated with a graduated alcohol-xylene series and coverslipped with cytoseal (Stephens Scientific, Riverdale, NJ, USA).

\section{Specificity of the antibodies}

Control experiments were carried out by incubating sections in the absence of the primary antibody, either calbindin or parvalbumin. No immunostaining was detected under these conditions. Further- more, the specificity of the antibodies used in the present study has been tested previously for several brains regions and also by Western blot (Vater and Braun, 1994; Chard et al., 1995; Henkel and Brunso-Bechtold, 1998; Martignoni et al., 2004).

\section{Densitometric analysis of calbindin and parvalbumin immunostaining}

The densitometric analysis method used in this study has been described in detail previously (Fuentes-Santamaria et al., 2003, 2005b; Alvarado et al., 2004, 2005). The analysis was performed in four equally spaced sections from the middle third of the nucleus in each animal, avoiding the rostral and caudal poles. In each section, three sampling fields (dorsal, middle and ventral) were defined in the deep layers in both control and epileptic animals. Selected fields for the quantification of calbindin and parvalbumin immunostaining were examined with brightfield illumination using a Leitz DMRB microscope with a $40 \times$ objective and images were captured under the same conditions by using a digital camera (Cohu CCD) attached to the microscope. After the color images were digitized, the red channel was used to obtain eight-bit images containing grayscale values from 0 (white) to 255 (black). Considering that the captured images could present slight variations due to the section thickness, non-uniformities in the light source, and irregularities in the microscope optics (Mize, 1985; Russ, 1990) that might affect the densitometric analysis, an unbiased normalization process, based on the signal-to-noise ratio principle (Herborn et al., 2002), was applied using the Scion Image software (Scion Corp., version beta 4.0.2). This procedure allows an adequate comparison and better visualization of the labeling in the SC in both control and epileptic animals (Fuentes-Santamaria et al., 2003).

A profile was defined as immunostained if the mean gray value was above that threshold for detection that was set as two standard deviations above the mean optical density of the whole image (Fuentes-Santamaria et al., 2003, 2005a,b; Alvarado et al., 2004, $2005,2007)$. Since it has been reported that immunostaining can be used as a relative measure of antigen levels (Huang et al., 1996; Yao and Godfrey, 1997), the mean gray level within the SC was used as an indirect indicator of the amount of either calbindin or parvalbumin in superficial and deep layers, respectively. In addition, given that changes in the mean gray level of immunostaining within each SC layer could be due either to changes in the immunostained neuropil and/or immunostained neurons, the mean gray level of the immunostained neuropil and the mean gray level within SC neurons were quantified as a measure of either calbindin or parvalbumin levels in the GPG/Vall hamster compared with control animals. Therefore, for comparison between control and epileptic groups, three quantitative indices were measured in calbindin and parvalbumin immunostained sections: (1) mean gray level of immunostaining within the nucleus, (2) mean gray level within neurons, and (3) mean gray level of the immunostained neuropil.

\section{Measurement of SC volume}

Using Neurolucida software (Microbrightfield, Colchester, VT), the perimeter of the $\mathrm{SC}$ in both control and epileptic animals was traced from every fourth cresyl-violet section throughout its rostrocaudal extent. The total volume of each SC was estimated from the compiled data using the Neuroexplorer component of Neurolucida. The mean volumes of the SC for both control and experimental groups were calculated and compared using a Student's $t$-test.

\section{Measurement of the cross-sectional area of SC neurons}

Cross-sectional areas of SC neurons in both GPG/Vall and control hamsters were measured from every fourth cresyl-violet section 
throughout the middle third of the nucleus using a $60 \times$-oil immersion objective. Only neurons with a well-defined cytoplasm, clear nuclear outline and a visible nucleolus were measured in Nisslstained sections. Quantification was performed using the software Scion Image for Windows.

\section{Measurement of amino acid levels in the SC}

\section{Sample preparation}

Control $(n=4)$ and epileptic $(n=4)$ adult male golden hamsters were decapitated with a small animal decapitator and without anesthesia. The animal head was quickly frozen on dry ice, the brain removed and the SC isolated from the frozen head. Since the duration of time between death and cessation of tissue biochemical activity has been reported to affect measured concentrations of some amino acids (Tews et al., 1963; Shank and Aprison, 1971; Balcom et al., 1975), the dissection of the SC was performed as quickly as possible (between 2 and $3 \mathrm{~min}$ ). Each tissue sample was weighed (approximately $20 \mathrm{mg}$ ), homogenized in $0.4 \mathrm{~N}$ perchloric acid, sonicated (Soniprep 150 MSE, Watford, UK), and centrifuged (Hettich Mod. EBA12, Prague, Czech Republic) at 15,000 rpm for $20 \mathrm{~min}$. The upper clear supernatants were then transferred into glass or plastic tubes inserts for HPLC measurement of amino acids.

\section{HPLC analysis}

For HPLC analysis, supernatants were neutralized with $0.1 \mathrm{~N}$ sodium hydroxide and derivatized with ortho-phthal-dialdehyde (OPA). The amino acids derivatization was performed following essentially the method described by Jones et al. (1981) with one modification. In the derivatization reaction, the 2-mecaptoethanol was substituted by 3-mercaptopropionic acid. This change improved the analysis sensitivity as much as two-fold (Herranz, personal communication). The method sensitivity was $1 \mathrm{pmol} / \mu \mathrm{l}$. The fluorescent derivatized amino acids were separated by reversed-phase chromatography on a Micra C18 column $(33 \mathrm{~mm} \times 4.6 \mathrm{~mm}$, particle size $1.5 \mathrm{~mm})$ using gradient elution. The mobile phase was comprised of solvent $A$ ( $0.05 \mathrm{M}$ sodium acetate; $\mathrm{pH} 5.88$ ) and solvent $\mathrm{B}$ (methanol) with a binary gradient. Ultra pure water and solvents were filtered through $0.20 \mu \mathrm{m}$ filters from Millipore (Bedford, MA, USA). The solvent flow rate was adjusted to $0.5 \mathrm{ml} / \mathrm{min}$ and the injection volume was $10 \mu \mathrm{l}$. Fluorescence detection (Perkin-Elmer LS4) was performed at 365 and $455 \mathrm{~nm}$ for excitation and emission wavelengths, respectively. Amino acids were identified by their retention times, and their con-
(A)

(C)

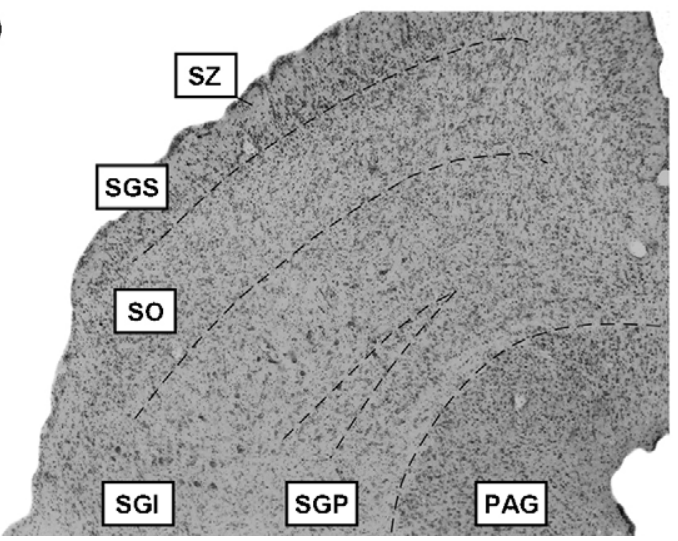

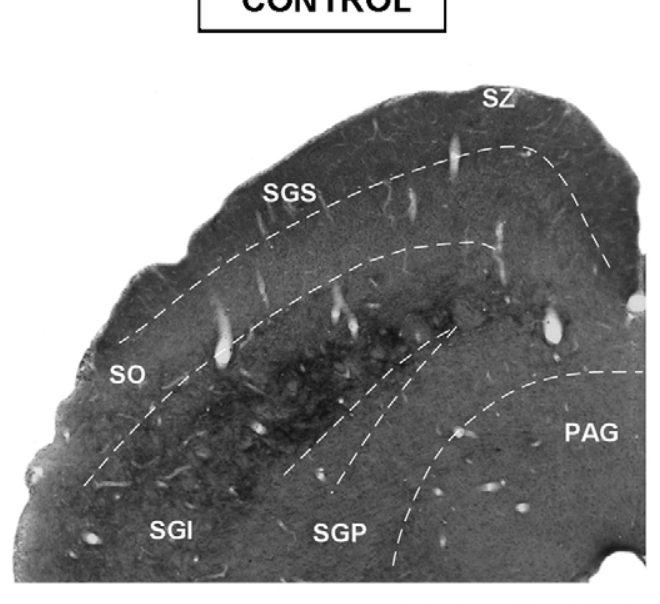

\section{CONTROL}

(B)

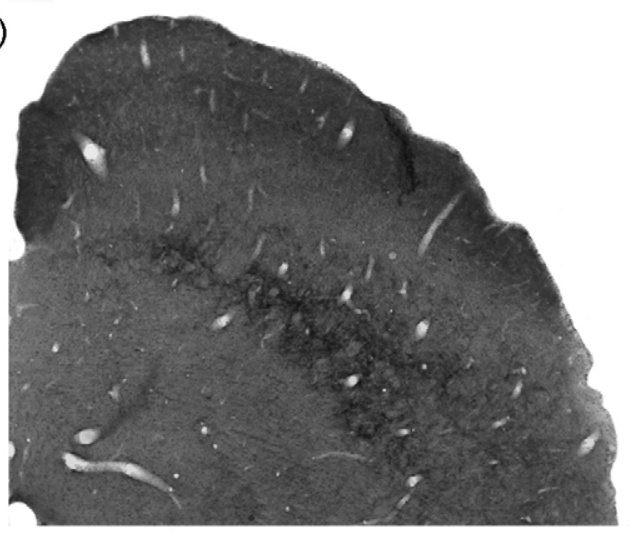

(D)

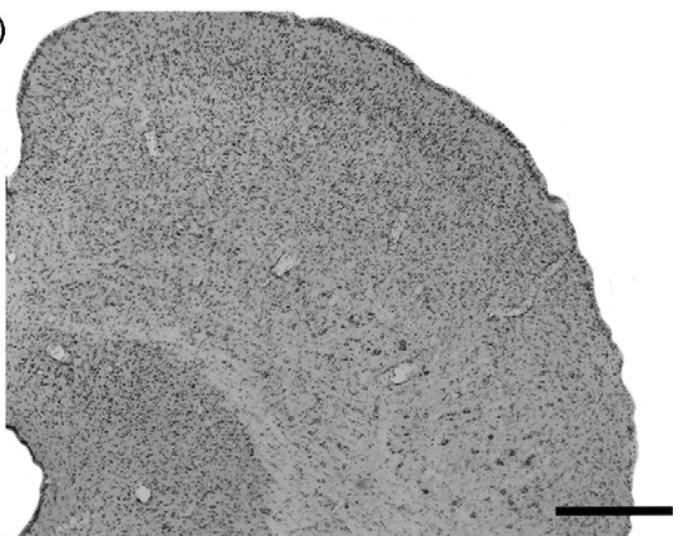

Figure 1 Low-magnification digital images showing $A C h E(A$ and $B)$ and cresyl-violet $(C$ and $D)$ stained coronal sections of the SC in control and epileptic animals. These stained sections were used to determine laminar boundaries according to stained fibers ( $A$ and $B$ ) and neurons ( $C$ and $D)$, respectively. Qualitatively, there was not an obvious decrease in size of the SC of the GPG/Vall hamster. However, measurement of SC volume revealed a significant decrease $(4.21 \pm 1.74 \%)$ in the size of the SC of epileptic animals compared to control hamsters. Dashed lines in A and C indicate the approximate borders between SC layers. Scale bar $=500 \mu \mathrm{m}$ in $\mathrm{D}$ (applies to A-D). Abbreviations: SZ, stratum zonale; SGS, stratum griseum superficiale; SO, stratum opticum; SGI, stratum griseum intermediale; SGP, stratum griseum profundum; PAG, periaqueductal gray. 

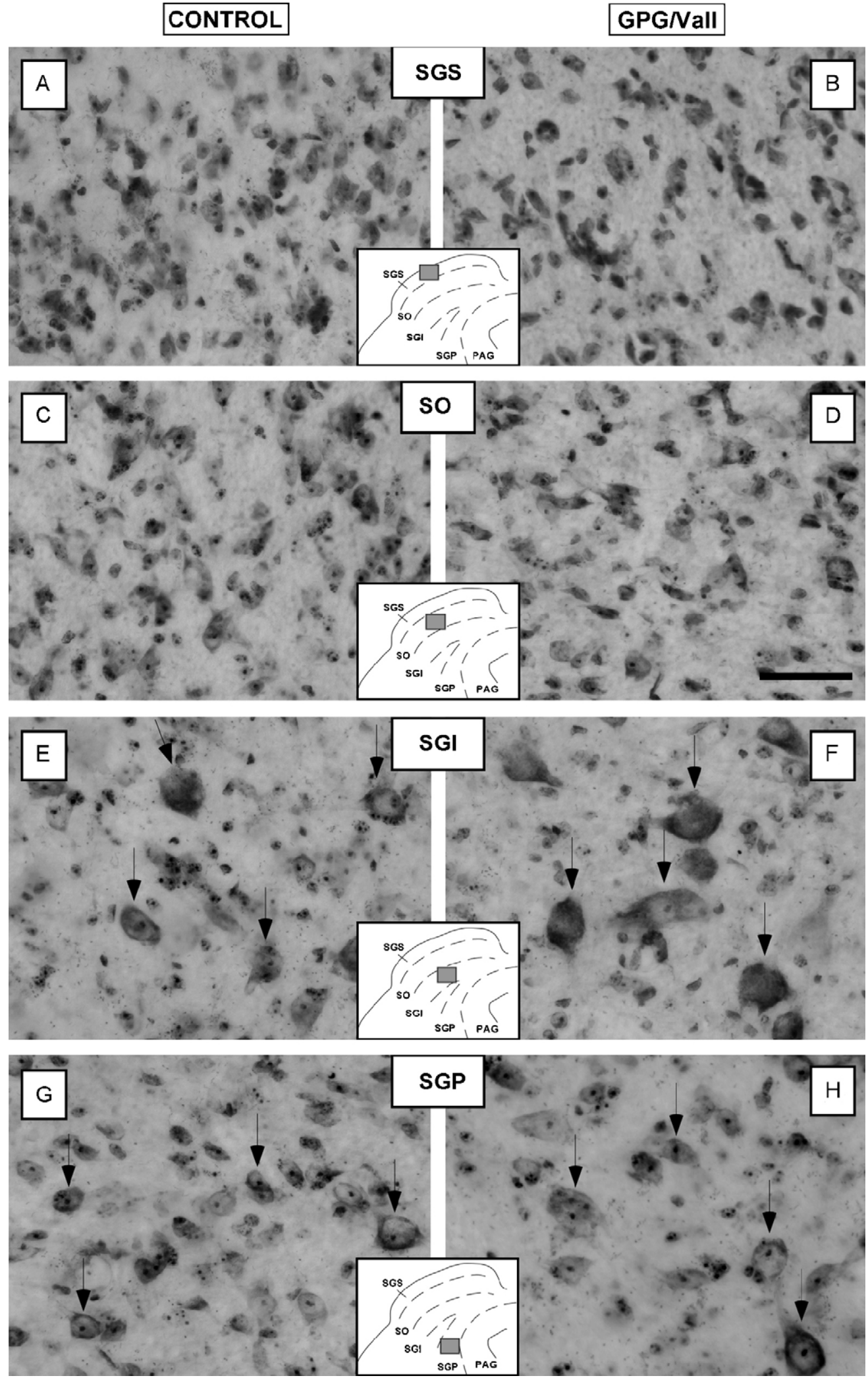

Figure 2 High-magnification digital images illustrating Nissl-staining in coronal sections of the SC in control (A, C, E and G) and GPG/Vall (B, D, F and H) hamsters. In comparison with control animals, there was an increase in the mean cross-sectional area of cresyl-violet stained neurons in the SGI (arrows in E and F) and SGP (arrows in G and H) of the GPG/Vall hamster but not in neurons in the SGS (A and $B)$ and SO $(C$ and $D)$. Gray square boxes in each drawing indicate the location of the higher magnification images shown in each panel. Scale bar $=50 \mu \mathrm{m}$ in D. Abbreviations: SZ, stratum zonale; SGS, stratum griseum superficiale; SO, stratum opticum; SGI, stratum griseum intermediale; SGP, stratum griseum profundum; PAG, periaqueductal gray. 
centrations were calculated by comparison to calibrated amino acid external standard solutions $(1.5 \mu \mathrm{M})$.

\section{Image processing}

To prepare the figures, the size and brightness of the images were adjusted using Adobe Photoshop (version 5.5) and Canvas (version 6.0). These adjustments in no way altered the immunostaining pattern of each antibody.

\section{Statistical analysis}

Immunocytochemical data were expressed as means \pm standard error while concentrations of each amino acid were as nmol/mg wet weight. Data were analyzed statistically using a Student's $t$-test. Statistical significance was determined at a level of $P<0.05$.

\section{Results}

\section{Morphology of the SC of the GPG/Vall hamster}

In order to delineate the patterning of the SC of control and epileptic animals, AChE and cresyl-violet stained sections were used to determine laminar boundaries according to stained fibers and neurons, respectively (Fig. 1A-D). Survey of sections stained with either $A C h E$ or cresyl violet did not show an obvious decrease in overall size of the SC of the GPG/Vall hamster compared to control animals (Fig. 1A-D). However, comparisons of volume measurements in coronal sections through comparable levels of the SC revealed a small but significant reduction $(4.21 \pm 1.74 \%)$ in the size of the SC of epileptic animals. In addition to changes in volume, the cross-sectional area of cresyl-violet stained neurons was examined in both control and GPG/Vall animals (Fig. 2). Accordingly, a statistically significant increase $(P<0.0001$ for the SGI and $P<0.05$ for the SGP) in the cross-sectional area of neurons located in the deep layers but not in the superficial layers of SC was observed in the GPG/Vall hamster compared to control animals (Fig. 3; also compare arrows

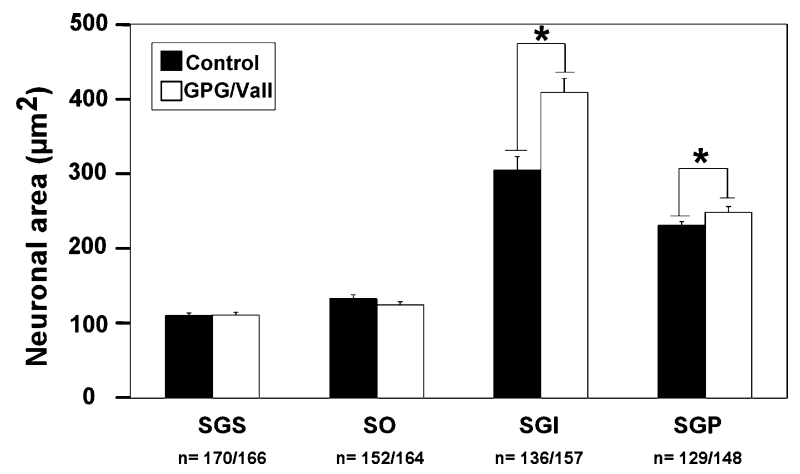

Figure 3 Bar graph showing the mean cross-sectional area of cresyl-violet stained neurons in the SC in control and GPG/Vall hamsters. A significant increase in the area of Nissl-stained neurons located in the deep layers (SGI and SGP) but not in the superficial layers was observed in the epileptic hamster compared to control animals. Values indicate mean, and error bars, standard errors. "Significant differences between groups $(P<0.05)$; $n$ indicates the number of neurons measured in control and epileptic animals. in Fig. $2 \mathrm{E}-\mathrm{F}$ and $\mathrm{G}-\mathrm{H}$ for the $\mathrm{SGI}$ and the SGP, respectively).

\section{Calbindin distribution in the SC of control and GPG/Vall hamsters}

In control animals, calbindin immunostaining was distributed in the superficial layers of the SC (Fig. 4A). Little calbindin immunostaining was observed however in the deeper layers, with the exception of a few scattered immunostained neurons in the SGI (Fig. 4A). Specifically, the SZ and the SGS presented a lightly immunostained neuropil and immunostained neurons that were small in size with round cell bodies and with dendritic processes that sometimes were not clearly visible (Fig. 4B; also see Fig. 5A). A similar pattern was also observed in the fibrous layer SO, although with a higher neuronal density (Fig. 4C). In general, neurons in this layer had stellate and fusiform morphologies and were darkly immunostained (arrows in Fig. 4C, also see Fig. 5B) although a few neurons immunostained lightly were also observed (Fig. 4C). Most of the neurons in the SO had immunostained dendritic fields that were either oriented upward toward the superficial layer or running in all directions (Fig. 4C). For comparison with epileptic animals, three quantitative indices of calbindin immunostaining were measured as described in Methods: (1) mean gray level within the nucleus, (2) mean gray level within neurons, and (3) mean gray level of the immunostained neuropil (Fig. 6).

Analysis of calbindin immunostaining in the SC of the GPG/Vall hamster did not show a different immunostaining pattern compared to control animals (Figs. 4 and 5). Quantitative measurements of the mean gray level throughout the SZ, SGS and SO, the mean gray level within neurons in these layers and the mean gray level of the immunostained neuropil revealed no significant differences between epileptic and control hamsters (Fig. 6).

\section{Parvalbumin distribution in the SC of control and GPG/Vall hamsters}

Parvalbumin immunostaining in the SC of control hamsters was distributed in a pattern that was complementary to the distribution of calbindin immunostaining (compare Figs. 4 and 7). As shown in Fig. 7A, the immunostaining within neurons and fibers was restricted to the deep layers, with the exception of a few scattered immunostained neurons in the SGS that will not be considered further here. No parvalbumin immunostaining was observed either in the SZ or in the SO. Within the SGI, although medium and large multipolar neurons lightly immunostained were the most abundant population (black arrows in Fig. 7B; also see Fig. 5C), small round immunostained neurons were also present (white arrowheads in Fig. 7B). Conversely in the SGP, small round immunostained neurons were most common (Fig. 7C; also see Fig. 5D). Notice that the neuropil in the SGI and SGP was darkly immunostained, particularly in the ventrolateral part of the nucleus (Fig. 7B and C).

Analysis of parvalbumin immunostaining in the deep layers of the SC of the GPG/Vall hamster showed a different pattern of immunostaining compared to control animals (compare Fig. 7D with Fig. 7A). Specifically, parvalbumin 
CONTROL
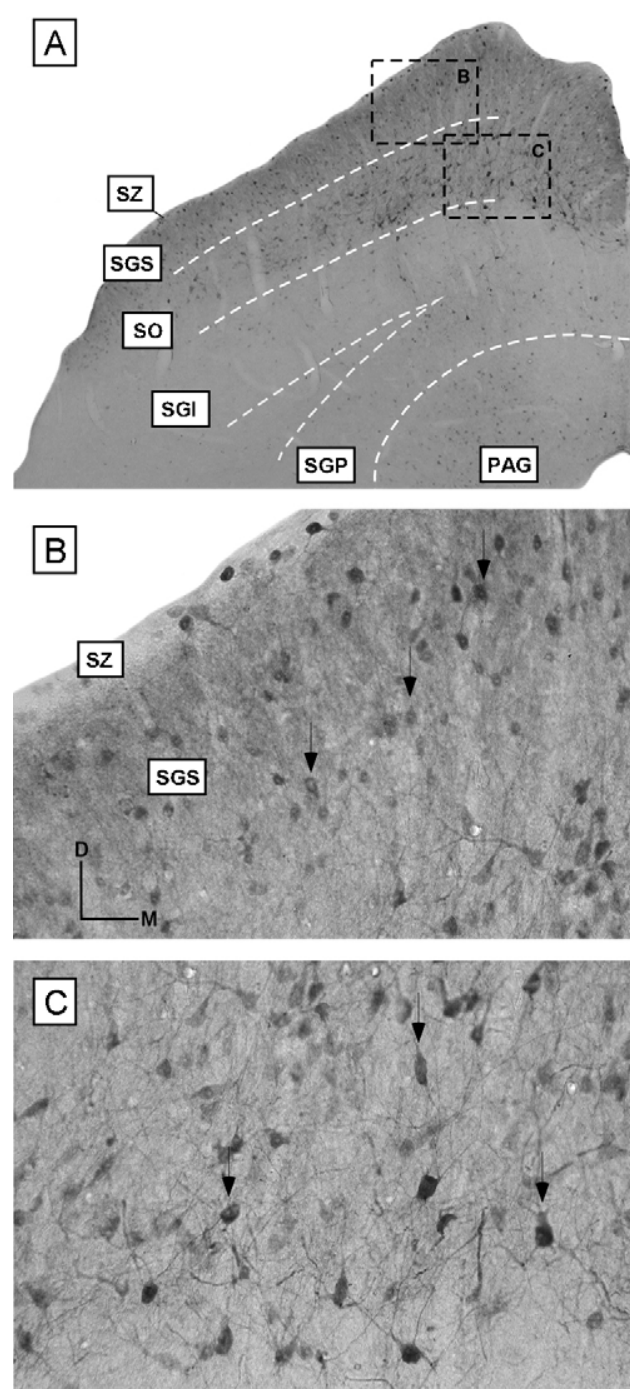
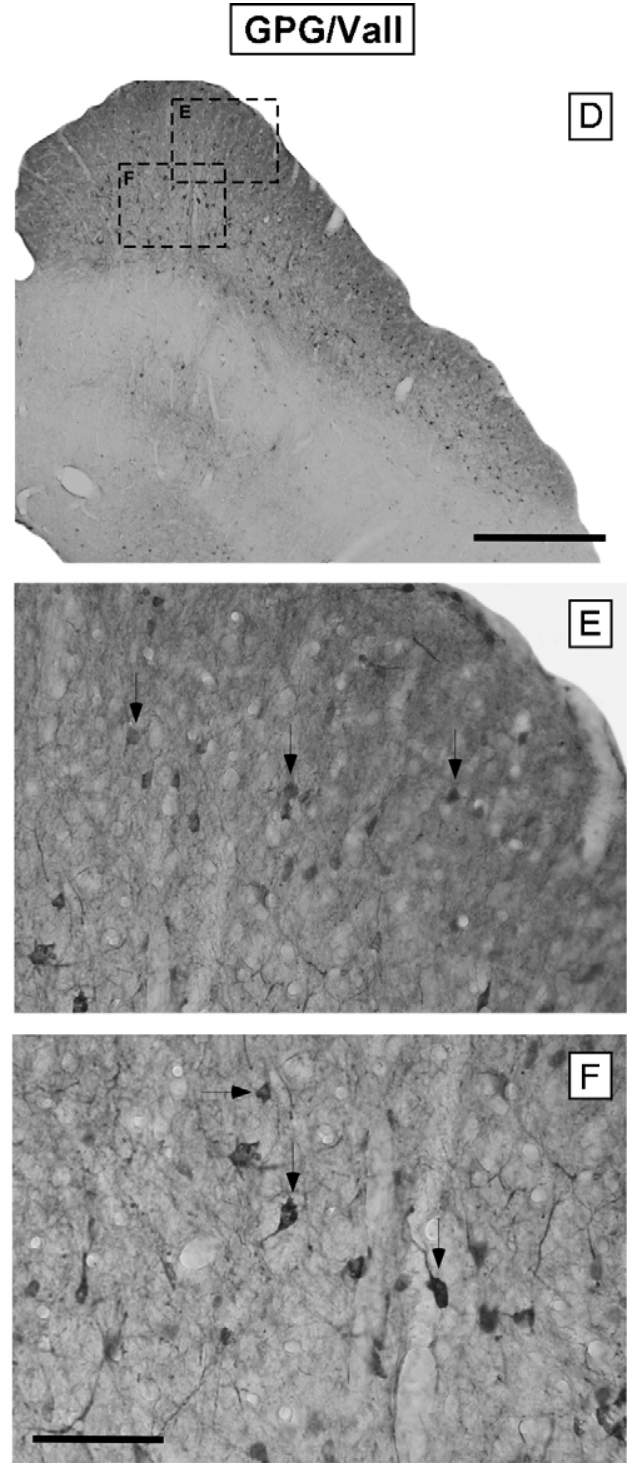

Figure 4 Digital images illustrating the distribution of calbindin immunostaining in the superficial layers of the SC in control (A-C) and epileptic (D-F) animals. No differences in the pattern of calbindin immunostaining were observed between groups. In the SZ and SGS, the immunostained neuropil as well as the immunostained neurons (arrows in B and E) were lightly immunostained in both control and epileptic animals. A similar pattern of distribution was observed in the SO, although neurons were more darkly immunostained (arrows in $\mathrm{C}$ and $\mathrm{F}$ ). Dashed white lines in A indicate the approximate borders between SC layers. Square boxes in $A$ and $B$ indicate the location of the higher images shown in $B$ and $C$ and $E$ and $F$, respectively. Scale bar $=500 \mu \mathrm{m}$ in $D$ (applies to $A$ and D), $100 \mu \mathrm{m}$ in F (applies to B, C, E and F). Abbreviations: SZ, stratum zonale; SGS, stratum griseum superficiale; SO, stratum opticum; SGI, stratum griseum intermediale; SGP, stratum griseum profundum; PAG, periaqueductal gray.

was detected mainly within somata that were mostly intermediate to large in size and were pyriform or round in shape (Fig. 5G-H). These immunostained neurons were darker and larger in size compared to control animals (Fig. 5, also compare Fig. 7E with Fig. 7B and Fig. 7F with Fig. 7C for the SGI and SGP, respectively). Conversely, the immunostained neuropil in the GPG/Vall hamster was decreased throughout the nucleus (compare Fig. 7D with Fig. 7A). Densitometric analysis of parvalbumin immunostaining throughout the deep layers of the SC in both groups indicated that despite a lack of change in the overall mean gray level, there was a significant increase $(P<0.0001$ for the SGI; $P<0.0001$ for the SGP) in the mean gray level within neurons as well as a significant decrease $(P<0.01$ for the SGI; $P<0.05$ for the SGP) in the immunostained neuropil in the deep layers of the GPG/Vall hamster compared to control animals (Fig. 8; also see Figs. 5 and 7).

\section{Measurement of amino acid levels in the SC}

In order to evaluate changes in amino acid levels that could be associated with seizure-susceptibility, the concentrations of aspartate, glutamate, glycine, taurine and GABA in the SC of control and epileptic animals were determined by HPLC. No differences between epileptic and control animals were observed in the levels of the excitatory neurotransmitters 


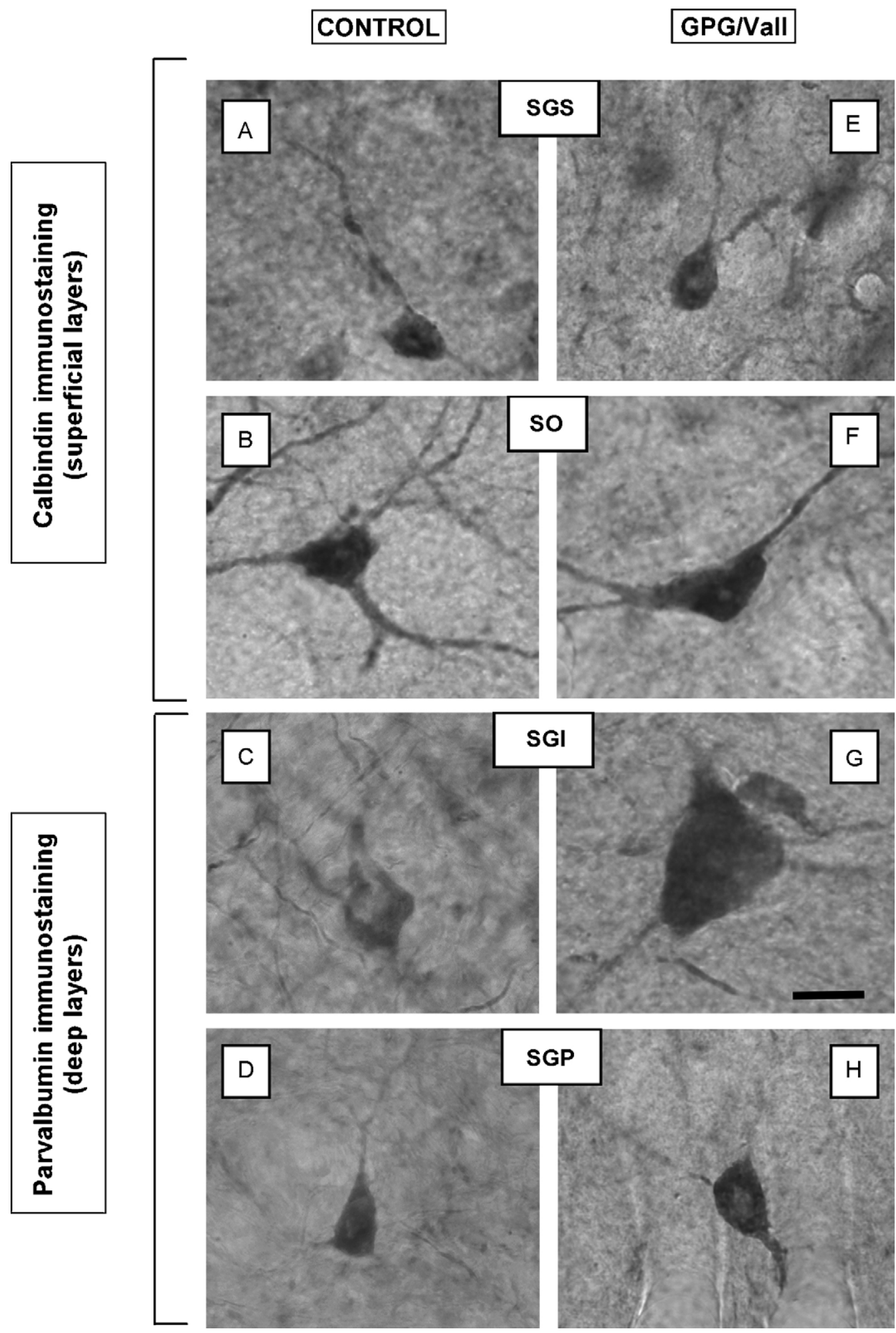

Figure 5 High-magnification digital images showing calbindin (A, B, E and F) and parvalbumin (C, D, G and H) immunostained neurons in the SC of control and epileptic hamsters. Calbindin immunostaining in the superficial layers was characterized by small and medium size neurons with dendritic processes oriented toward the surface of the nucleus (A and E). In the fibrous layer SO, stellate $(B)$ and fusiform $(F)$ neurons of medium size were frequently observed. Conversely, parvalbumin immunostaining in the deeper layers was characterized by large and intermediate multipolar neurons with proximal dendrites clearly visible (C, D, G and $\mathrm{H})$. Notice that immunostained neurons in the deeper layers of the GPG/Vall hamster are larger and more darkly immunostained that those in control animals (compare $\mathrm{G}$ with $\mathrm{C}$ and $\mathrm{H}$ with D for the SGI and SGP, respectively). Scale bar $=25 \mu \mathrm{m}$ in $\mathrm{G}$. Abbreviations: SZ, stratum zonale; SGS, stratum griseum superficiale; SO, stratum opticum; SGI, stratum griseum intermediale; SGP, stratum griseum profundum; PAG, periaqueductal gray. 

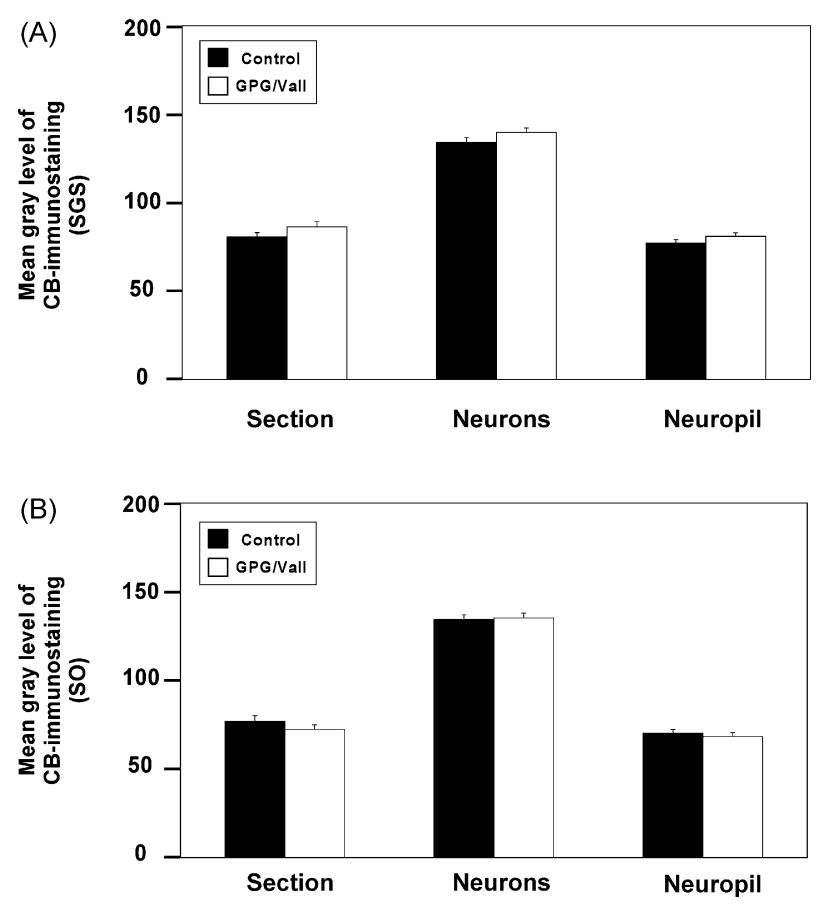

Figure 6 Bar graphs showing the mean gray level of calbindin immunostaining in the superficial layers of the SC in control and GPG/Vall hamsters. Quantitative measurements of the mean gray level throughout the SGS (A) and SO (B), the mean gray level within neurons in these layers and the mean gray level of the immunostained neuropil revealed no significant differences between epileptic and control hamsters. Values indicate mean, and error bars, standard errors.

aspartate and glutamate (Table 1). Conversely, analysis of the inhibitory system in the SC showed a decrease in GABA levels as well as an increase in taurine concentration without changes in glycine levels in the GPG/Vall hamster compared to control animals (Table 1 ).

\section{Discussion}

In the present study, morphologic and neurochemical abnormalities in the deep layers of the SC of the GPG/Vall hamster that might be associated with seizure susceptibility were investigated by using immunocytochemical, densitometric analysis and HPLC. Analysis of Nissl-stained sections revealed a small but significant reduction in the $\mathrm{SC}$ volume in the GPG/Vall hamster compared to control animals. This decrease was concomitant with a significant increase in the cross-sectional area of cresyl-violet stained neurons located in the deep but not in the superficial layers of the SC. Quantitative analysis of calbindin immunostaining (immunostained fibers and neurons) in the superficial layers of the SC did not show significant differences between epileptic and control animals. Conversely, analysis of parvalbumin immunostaining in the deep layers of the GPG/Vall hamster revealed a significant increase in the mean gray level of parvalbumin immunostaining within neurons as well as a decreased immunostained neuropil compared to control hamsters. In addition to these changes, amino acid analysis of the SC of the epileptic hamster with HPLC revealed a significant decrease in the levels of GABA and increased levels of taurine without significant changes in glutamate, aspartate and glycine levels compared to control hamsters.

Several studies have evaluated the contribution of the SC in the neuronal network for audiogenic seizures in a variety of audiogenic seizure-susceptible animals (Willott and Lu, 1980; Cools et al., 1984; Millan et al., 1986; Ribak et al., 1994, 1997; Faingold, 1999; Faingold and Casebeer, 1999; Faingold and Randall, 1999). Accordingly, in situ hybridization studies for c-fos-mRNA in GEPR-9s have shown a dense labeling in the intermediate and deep layers of the SC after AGS, providing evidence that this nucleus is involved in the propagation of seizure activity (Ribak et al., 1997). Similarly, the role of the SC of GEPR-9s in seizure progression is supported by reduction of the audiogenic response score after bilateral knife cuts (Ribak et al., 1994). Faingold and Randall (1999) examined the firing of SC neurons located in the deep layers of the SC as well as the convulsive behavior in freely moving GEPRs with chronically implanted electrodes and observed that these neurons exhibited a rapid burst firing that precedes the wild running phase of AGS. This intense firing pattern may be due to an increased input to the deep layers of the SC from auditory nuclei as well as to alterations in inhibitory and excitatory amino acids (Faingold and Randall, 1999). Similar to GEPRs, the present study also suggests that the SC of the GPG/Vall hamster is implicated in the neuronal network of AGS. In particular, a reduction in the volume of the SC of the epileptic hamster has been observed compared to control animals. Decreases in volume have been associated with structural damage not only in animal models of AGS (Fuentes-Santamaria et al., 2005a), but also in different brain regions of patients having different types of epilepsies (Barr et al., 1997; Lawson et al., 2000; Bernasconi et al., 2001). The present findings also report a significant increase in the mean cross-sectional area of neurons located in the deep layers of the SC of the epileptic hamster compared to control animals which is consistent with alterations in presumptive output neurons projecting to

Table 1 Amino acid levels in the superior colliculus in control and GPG/Vall hamsters

\begin{tabular}{|c|c|c|c|c|c|}
\hline & \multicolumn{2}{|l|}{ Excitatory } & \multicolumn{3}{|l|}{ Inhibitory } \\
\hline & Aspartate & Glutamate & Glycine & Taurine & GABA \\
\hline Control $(n=4)$ & $3.09 \pm 0.03$ & $3.12 \pm 0.05$ & $1.42 \pm 0.05$ & $1.79 \pm 0.04(*)$ & $3.12 \pm 0.01\left(^{*}\right)$ \\
\hline GPG/Vall $(n=4)$ & $3.01 \pm 0.02$ & $3.19 \pm 0.01$ & $1.23 \pm 0.05$ & $2.36 \pm 0.05(*)$ & $2.59 \pm 0.01()^{*}$ \\
\hline
\end{tabular}

Values expressed as nmol/mg wwt, are means and standard error. Asterisk ( ${ }^{*}$ ) indicates significant differences between control and GPG/Vall hamsters $(P<0.05)$. 

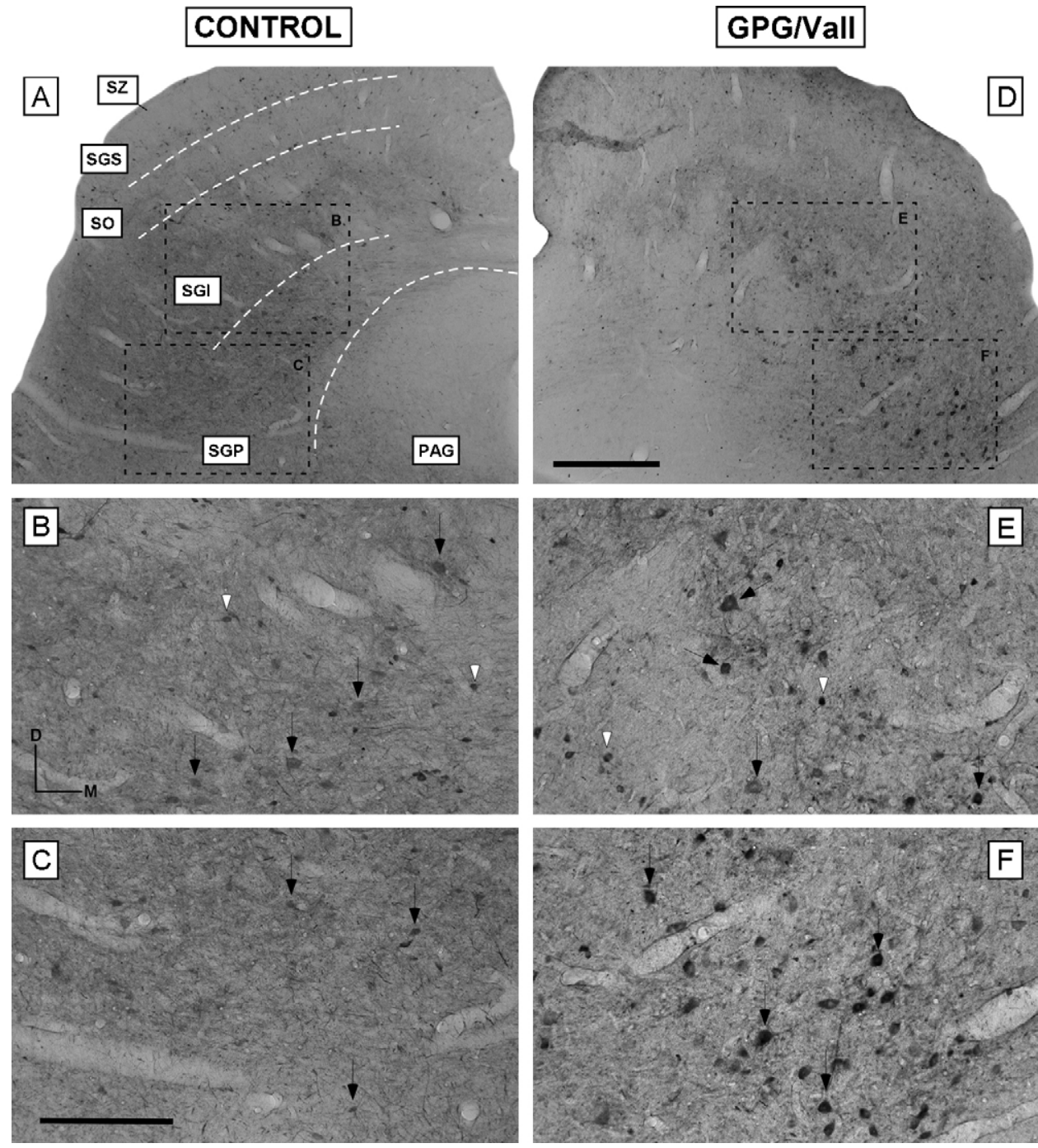

Figure 7 Digital images illustrating the distribution of parvalbumin immunostaining in the deep layers of the SC in control (A-C) and epileptic (D-F) animals. Although within the SGI and SGP of both groups, medium and large multipolar neurons were the most common population, small neurons were also observed (compare arrows with arrowheads in B and $\mathrm{E}$, and $\mathrm{C}$ and $\mathrm{F}$ for the SGI and SGP, respectively). In the GPG/Vall hamster, while the immunostained neuropil was decreased, the immunostained neurons in the deep layers (SGI and SGP) were darker and larger in size compared to control animals (compare arrows in E and F with arrows in $B$ and C, for the SGI and SGP, respectively). Dashed white lines in A indicate the approximate borders between SC layers. Square boxes in $A$ and $B$ indicate the location of the higher images shown in B, C, E and F. Scale bar $=500 \mu \mathrm{m}$ in $D$ (applies to $A$ and $D$ ); $250 \mu \mathrm{m}$ in C (applies to B, C, E and F). Abbreviations: SZ, stratum zonale; SGS, stratum griseum superficiale; SO, stratum opticum; SGI, stratum griseum intermediale; SGP, stratum griseum profundum; PAG, periaqueductal gray.

premotor and motor centers. Neuronal hypertrophy also has been reported for spiral ganglion cells in the GPG/Vall hamster (Fuentes-Santamaria et al., 2005a) and also for neurons in the neocortex of patients with temporal lobe epilepsy (Bothwell et al., 2001). Although the functional significance of this hypertrophy is not clear, it has been hypothesized that it may represent an increased metabolic support necessary for the new growth of synaptic endings that might be replacing degenerating axons (Bothwell et al., 2001) due to brain damage in epileptic patients (Houser, 1992; Du et al., 1993). Consistent with this hypothesis, the atrophic changes reported in the ascending auditory pathway in the GPG/Vall hamster (Fuentes-Santamaria et al., 2005a) could lead to a reduction in synapses in the deep layers of the SC. Such atrophy would induce sprouting of new terminals that consequently, may increase the excitatory flux into SC neurons and as a result, the likelihood of epileptic seizures (Bothwell et al., 2001).

Epileptic seizures have been shown to affect not only excitability but also neuronal viability and intracellular signaling pathways in which calcium-related mechanisms are involved (Palayoor and Seyfried, 1984). Calcium-binding proteins contribute to regulation of intracellular calcium concentration in neurons in the nervous system (Baimbridge et al., 1992). Interestingly, it has been suggested that intracellular calcium, and therefore, calcium-binding protein levels, could be regulated by modifications in neuronal electrical activity (Braun et al., 1991; Winsky and Jacobowitz, 


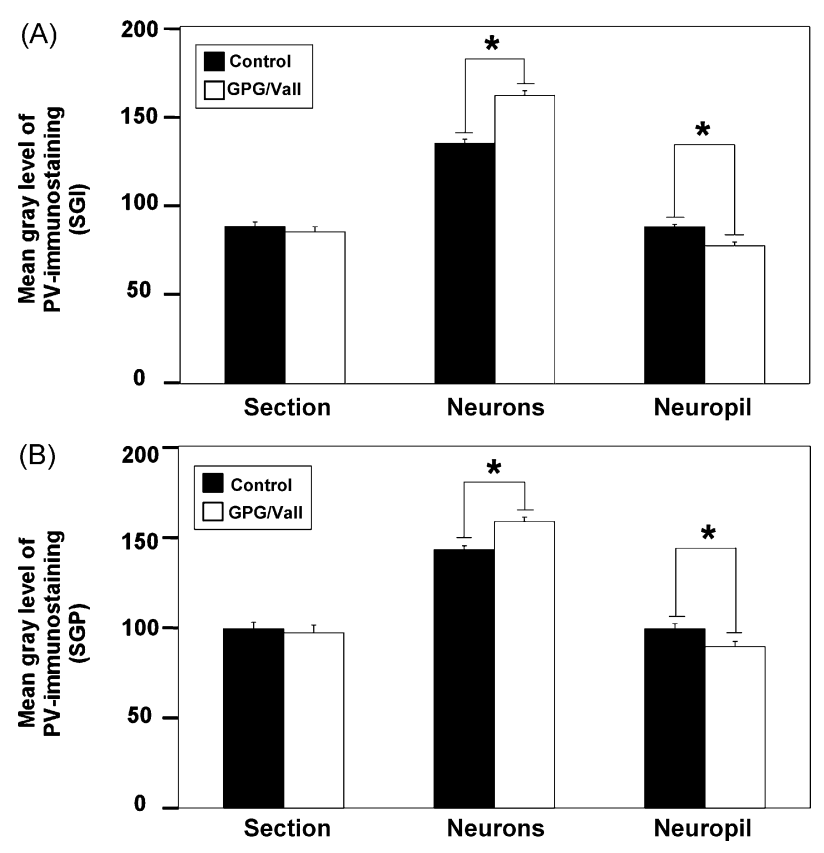

Figure 8 Bar graphs showing the mean gray level of parvalbumin immunostaining in the deep layers of the SC in control and GPG/Vall hamsters. Quantitative analyses of the mean gray level of parvalbumin immunostaining in the SGI (A) and SGP (B) revealed a significant increase in mean gray level within neurons as well as a significant decrease in the immunostained neuropil in the deeper layers of the epileptic hamster compared to control animals (A and B). Values indicate mean, and errors bars, standard errors. "Significant differences between groups $(P<0.05)$.

1995; Fuentes-Santamaria et al., 2003, 2005b; Alvarado et al., 2004, 2005). One of these proteins, calbindin, has been used in the present study as a neuronal marker for investigating possible activity-related changes in the superficial layers of the SC of the GPG/Vall hamster. No changes in calbindin immunostaining either within neurons or fibers were observed in the superficial layers of the SC in the GPG/Vall hamster compared to control animals (Behan et al., 1992; Park et al., 2004), suggesting that levels of this protein are probably not modulated by changes in activity during AGS.

Conversely to calbindin, parvalbumin is a useful marker of neurons with high levels of electrical and metabolic activity (Braun et al., 1985, 1991; Kawaguchi et al., 1987). This protein labels efferent neurons whose axons exit the SC via the tectoreticular pathway en route to the contralateral brainstem (Meredith and Stein, 1986; Wallace et al., 1993; McHaffie et al., 2001; Fuentes-Santamaria et al., 2006), which is consistent with the present study reporting parvalbumin within intermediate and large multipolar neurons in the deep layers of the SC in control and epileptic animals. An increase in the mean gray level of parvalbumin immunostaining within neurons as well as a decreased immunostained neuropil have been found in the deep layers of the SC of the GPG/Vall compared to control animals. Upregulation in the levels of calcium-binding proteins has been reported in the IC after sound stimulation (Idrizbegovic et al., 1999) and cochlear ablation (Fuentes-Santamaria et al., 2003; Alvarado et al., 2005), suggesting that calcium-buffering capacity is regulated by neuronal activity. Therefore, it might be expected that increased excitability as a consequence of downregulation in inhibition (McAlpine et al., 1997; Mossop et al., 2000; Salvi et al., 2000) could lead to increased calcium-binding protein levels within auditory neurons. Accordingly, increased levels of parvalbumin immunostaining in the IC and also in its afferent sources have been observed in the GPG/Vall hamster (Fuentes-Santamaria et al., 2005a). This upregulation may be the result of an attempt to attenuate degeneration and therefore improve cell survival (Beers et al., 2001). Thus, it is possible that the significant decrease in the parvalbumin immunostained neuropil observed in the SC of the GPG/Vall hamster indicates an atrophy of neuronal processes such as dendrites while the significant increase in the mean gray level within parvalbumin immunostained neurons represents a neuroprotective effect against calcium overload associated with epileptic seizures (Kamphuis et al., 1989; Fabene et al., 2001). This upregulation may also reflect abnormalities in other calcium-related processes such as altered voltagedependent calcium channel currents (Burgess and Noebels, 1999).

The evidence for the involvement of GABA in the $S C$ in seizures comes from pharmacologic studies demonstrating that focal microinjections of GABA antagonists into the deep SC of normal rats can generate spontaneous seizure activity (Millan et al., 1986) and also wild running behavior (Cools et al., 1984). In particular in the IC of several genetic models of epilepsy, a decreased effectiveness of inhibition has been suggested to be one of the most important mechanisms modulating AGS susceptibility (see Faingold, 2002 for review; also see De Cabo de la Vega et al., 2006). Such a decrease has been proposed to be the result of either a genetically induced decrease in postsynaptic sensitivity to GABA or a compensatory mechanism for the hearing deficit in these animals (Faingold et al., 1986). In the present study, we found a significant decrease in the levels of GABA in the GPG/Vall hamster compared to control animals, suggesting an important role for this amino acid in the mechanism of seizure activity in the SC.

It is well-known that the duration of time between death and cessation of tissue biochemical activity affects measured concentrations of some amino acids (Shank and Aprison, 1971; Geddes et al., 1999). In particular, a rapid postmortem increase in GABA concentration has been reported to occur in the central nervous system (Shank and Aprison, 1971). Thus, it is possible that the levels of GABA reported in the present study could be due to postmortem changes in this amino acid. However, although some of these changes may occur, the fact that both groups of animals were processed simultaneously and under similar conditions, suggests that the significant differences observed between groups are indeed reflecting a decrease in GABA concentration in the epileptic hamster compared to control animals. In agreement with this finding, a similar decrease in GABA concentration has also been reported in several brain regions in seizure-naive and seizure-experienced GEPRs (Lasley, 1991) and also in mice after electroshock-induced epilepsy (De Luca et al., 2004). In contrast with the decreased levels of GABA in the SC described in the present study, Ribak et al. (1988) reported an increase in the concentration of GABA in the CNIC neurons in GEPRs compared to 
seizure-resistant animals as well as an increased number of GAD-immunostained neurons in this brain region (Roberts et al., 1985). The discrepancy between this latter study and our findings can be resolved by consideration of the different brain regions analyzed (CNIC versus SC) and it may also reflect species differences. In addition, in the present study, a significant increase in the levels of taurine without significant changes in glutamate, aspartate and glycine levels was also observed in the SC of the GPG/Vall animals as compared to control hamsters. Taurine is an inhibitory neuromodulator with anticonvulsant effects that has been proposed to enhance glutamate metabolism in GEPRs (Bonhaus and Huxtable, 1985; Ribak et al., 1988; Lasley, 1991). In agreement with these studies, increases in taurine concentration have been observed in the IC of GEPRs, suggesting an important role of this amino acid in the mechanism of seizure activity (Ribak et al., 1988; Lasley, 1991). Thus, considering the anticonvulsant effect of this amino acid in different brain regions, increases in taurine levels may reflect an epilepsy-dependent compensatory mechanism in response to an abnormal GABAergic system (Lasley, 1991).

Taken together, the present findings are consistent with the conclusions of previous studies reporting neurochemical and electrophysiological abnormalities in the SC of epilepsyprone animals (Willott and Lu, 1980; Cools et al., 1984; Millan et al., 1986; Ribak et al., 1994, 1997; Faingold, 1999). Specifically, our data report a hypertrophy of neurons located in the deep layers of the SC of the GPG/Vall hamster together with an upregulation in the mean gray level of parvalbumin immunostaining within these neurons. These increases both in surface area and parvalbumin levels may reflect a response to increased synaptic inputs that may result from an altered inhibitory function. Whether the abnormalities of the SC observed in the present study contribute to or are a consequence of the repeated seizures is not known and will require further study. However, these findings support the studies in other species showing that the deep layers of the SC are an important component of the audiogenic seizure network. Thus, implicating the SC in yet another species strengthens further the hypothesis that the $\mathrm{SC}$ is universally involved in audiogenic seizures.

\section{Acknowledgements}

The authors thank Dr. Anna R. Taylor for helpful comments on the manuscript and also to Dr. Purificacion Corchete for help with the sample preparation for the measurement of amino acid levels and to María José Asensio for technical assistance in the HPLC analysis. This study was supported by the Spanish Grants MEC\#BFU2006-00811, JCyL/FSE\#SA$007 C 05$ and SAN191/SA24/06.

\section{References}

Alvarado, J.C., Fuentes-Santamaria, V., Brunso-Bechtold, J., Henkel, C.K., 2004. Alterations in calretinin immunostaining in the ferret superior olivary complex after cochlear ablation. J. Comp. Neurol. 470, 63-79.

Alvarado, J.C., Fuentes-Santamaria, V., Franklin, S.R., BrunsoBechtold, J.K., Henkel, C.K., 2005. Unilateral cochlear ablation in adult ferrets results in upregulation in calretinin immunostain- ing in the central nucleus of the inferior colliculus. Neuroscience 136, 957-969.

Alvarado, J.C., Fuentes-Santamaria, V., Franklin, S.R., BrunsoBechtold, J.K., Henkel, C.K., 2007. Synaptophysin and insulinlike growth factor-1 immunostaining in the central nucleus of the inferior colliculus in adult ferrets following unilateral cochlear removal: a densitometric analysis. Synapse 61 (5), 288-302.

Baimbridge, K.G., Celio, M.R., Rogers, J.H., 1992. Calcium-binding proteins in the nervous system. Trends Neurosci. 15, 303-308.

Balcom, G.J., Lenox, R.H., Meyerhoff, J.L., 1975. Regional gamma-aminobutyric acid levels in rat brain determined after microwave fixation. J. Neurochem. 24, 609-613.

Barr, W.B., Ashtari, M., Schaul, N., 1997. Bilateral reductions in hippocampal volume in adults with epilepsy and a history of febrile seizures. J. Neurol. Neurosurg. Psychiatry 63, 461-467.

Beers, D.R., Ho, B.K., Siklos, L., Alexianu, M.E., Mosier, D.R., Mohamed, A.H., Otsuka, Y., Kozovska, M.E., McAlhany, R.E., Smith, R.G., Appel, S.H., 2001. Parvalbumin overexpression alters immune-mediated increases in intracellular calcium, and delays disease onset in a transgenic model of familiar amyotrophic lateral sclerosis. J. Neurochem. 79, 499-509.

Behan, M., Jourdain, A., Bray, G.M., 1992. Calcium binding protein (calbindin D28k) immunoreactivity in the hamster superior colliculus: ultrastructure and lack of co-localization with GABA. Exp. Brain Res. 89, 115-124.

Bernasconi, N., Bernasconi, A., Caramanos, Z., Dubeau, F., Richardson, J., Andermann, F., Arnold, D.L., 2001. Entorhinal cortex atrophy in epilepsy patients exhibiting normal hippocampus volumes. Neurology 56, 1335-1339.

Bonhaus, D.W., Huxtable, R.J., 1985. The interaction of taurine and glutamate metabolism in brains of genetically seizure susceptible and seizure resistant rats. Proc. West. Pharmacol. Soc. 28 , 103-105.

Bothwell, S., Meredith, G.E., Philips, J., Staunton, H., Doherty, C., Grigorenko, E., Glazier, S., Deadwyler, S.A., O’Donovan, C.A., Farrel, M., 2001. Neuronal hypertrophy in the neocortex of patients with temporal lobe epilepsy. J. Neurosci. 21, 4789-4800.

Braun, K., Scheich, H., Schachner, M., Heizmann, C.W., 1985. Distribution of parvalbumin, cytochrome oxidase activity and $\left[{ }^{14} \mathrm{C}\right] 2$-deoxyglucose uptake in the brain of the zebra finch, I. Auditory and vocal motor systems. Cell. Tissue Res. 240, 101-115.

Braun, K., Scheich, H., Heizmann, C.W., Hunziker, W., 1991. Parvalbumin and calbindin-D28K immunoreactivity as developmental markers of auditory and vocal motor nuclei of the zebra finch. Neuroscience 3, 853-869.

Browning, R.A., 1986. Neuroanatomical localization of structures responsible for seizures in the GEPR: lesion studies. Life Sci. 39, 857-867.

Burgess, D.L., Noebels, J.L., 1999. Single gene defects in mice: the role of voltage-dependent calcium channels in absence models. Epilepsy Res. 36, 111-122.

Chard, P.S., Jordan, J., Marcuccilli, C.J., Miller, R.J., Leiden, J.M., Roos, R.P., Ghadge, G.D., 1995. Regulation of excitatory transmission at hippocampal synapses by calbindin D28k. Proc. Natl. Acad. Sci. U.S.A. 92, 5144-5148.

Chakravarty, D.N., Faingold, C.L., 1996. Increased responsiveness and failure of habituation in neurons of the external nucleus of inferior colliculus associated with audiogenic seizures of the genetically epilepsy-prone rat. Exp. Neurol. 141, 280-286.

Chevalier, G., Mana, S., 2000. Honeycomb-like structure of the intermediate layers of the rat superior colliculus, with additional observations in several other mammals: AChE patterning. J. Comp. Neurol. 419, 137-153.

Coleman, J.R., Clerici, W.J., 1987. Sources of projections to subdivisions of the inferior colliculus in the rat. J. Comp. Neurol. 262, 215-226. 
Cools, A.R., Coolen, J.M., Smit, J.C., Ellenbroek, B.A., 1984 The striato-nigro-collicular pathway and explosive running behaviour: functional interaction between neostriatal dopamine and collicular GABA. Eur. J. Pharmacol. 100, 71-77.

De Cabo de la Vega, C., Villanueva-Hernandez, P., Prieto-Martin, A., 2006. The neurochemistry of epilepsy, inhibitory neurotransmission and experimental models: new perspectives. Rev. Neurol. 42, 159-168.

De Luca, G., Di Giorgio, R.M., Macaione, S., Calpona, P.R., Costantino, S., Di Paola, E.D., De Sarro, A., Ciliberto, G., De Sarro, G., 2004. Susceptibility to audiogenic seizure and neurotransmitter amino acid levels in different brain areas of IL-6-deficient mice. Pharmacol. Biochem. Behav. 78, 75-81.

Du, F., Whetsell, W., Abou-Khalil, B., Blumenkopf, B., Lothman, E., Schwarcz, R., 1993. Preferential neuronal loss in layer III of the entorhinal cortex in patients with temporal lobe epilepsy. Epilepsy Res. 16, 223-233.

Fabene, P.F., Correia, L., Carvalho, R.A., Cavalheiro, E.A., Bentivoglio, M., 2001. The spiny rat Proechimys Guyannensis as model of resistance to epilepsy: chemical characterization of hippocampal cell populations and pilocarpine-induced changes. Neuroscience 104, 979-1002.

Faingold, C.L., Travis, M.A., Gehlbach, G., Hoffman, W.E., Jobe, P.C., Laird, H.E., Caspary, D.M., 1986. Neuronal response abnormalities in the inferior colliculus of the genetically epilepsy-prone rat. Electroencephalogr. Clin. Neurophysiol. 63, 296-305.

Faingold, C.L., 1999. Neuronal networks in the genetically epilepsyprone rat. Adv. Neurol. 79, 311-321.

Faingold, C.L., Randall, M.E., 1999. Neurons in the deep layers of superior colliculus play a critical role in the neuronal network for audiogenic seizures: mechanisms for production of wild running behavior. Brain Res. 815, 250-258.

Faingold, C.L., Casebeer, D., 1999. Modulation of the audiogenic seizure network by noradrenergic and glutamatergic receptors of the deep layers of superior colliculus. Brain Res. 821, 392-399.

Faingold, C.L., 2002. Role of GABA abnormalities in the inferior colliculus pathophysiology-audiogenic seizures. Hear. Res. 168, 223-237.

Fuentes-Santamaria, V., Alvarado, J.C., Brunso-Bechtold, J.K., Henkel, C.K., 2003. Upregulation of calretinin immunostaining in the ferret inferior colliculus after cochlear ablation. J. Comp. Neurol. 460, 585-596.

Fuentes-Santamaria, V., Cantos, R., Alvarado, J.C., GarcíaAtares, N., López, D.E., 2005a. Morphologic and neurochemical abnormalities in the auditory brainstem of the genetically epilepsy-prone hamster (GPG/Vall). Epilepsia 46, 10271045.

Fuentes-Santamaria, V., Alvarado, J.C., Taylor, A.R., BrunsoBechtold, J.K., Henkel, C.K., 2005b. Quantitative changes in calretinin immunostaining in the cochlear nuclei after unilateral cochlear removal in young ferrets. J. Comp. Neurol. 483, 458-475.

Fuentes-Santamaria, V., Stein, B.E., McHaffie, J.G., 2006. Neurofilament proteins are preferentially expressed in descending output neurons of the cat the superior colliculus: a study using SMI- 32 . Neuroscience 138, 55-68.

Garcia-Cairasco, N., Terra, V.C., Doretto, M.C., 1993. Midbrain substrates of audiogenic seizures in rats. Behav. Brain Res. 58, 57-67.

Geddes, J.W., Chang, N.G., Ackley, D.C., Soultanian, N.S., McGillis, J.P., Yokel, R.A., 1999. Postmortem elevation in extracellular glutamate in the rat hippocampus when brain temperature is maintained at physiological levels: implications for the use of human brain autopsy tissues. Brain Res. 831 (1-2), 104-112.

Gil-Verona, J.A., Gómez, M.E., Gómez, P., Macías, J., GarcíaAtarés, N., 1991. Comportamiento morfológico de la porción troncoencefálica del sistema acústico en hámsters que padecen epilépsia audiógena. Rev. Esp. Epilepsia 6, 85-95.

Guitton, D., Munoz, D.P., 1991. Control of orienting gaze shifts by the tectoreticulospinal system in the head-free cat. I. Identification, localization, and effects of behavior on sensory responses. J. Neurophysiol. 66, 1605-1623.

Henkel, C.K., Brunso-Bechtold, J.K., 1998. Calcium-binding proteins and GABA reveal spatial segregation of cell types within the developing lateral superior olivary nucleus of the ferret. Microsc. Res. Tech. 41, 234-245.

Herborn, C.U., Waldschuetz, R., Lauenstein, T.C., Goyen, M., Lauffer, R.B., Moeroey, T., Debatin, J.F., Ruehm, S.G., 2002. Contrast-enhanced magnetic resonance imaging (MS-325) in a murine model of systemic lupus erythematosus. Invest. Radiol. 37, 464-469.

Houser, C.R., 1992. Morphological changes in the dentate gyrus in human temporal lobe epilepsy. Epilepsy Res. (Suppl. 7), 223-234.

Huang, X., Chen, S., Tietz, E., 1996. Immunocytochemical detection of regional protein changes in rat brain sections using computerassisted image analysis. J. Histochem. Cytochem. 44, 981-987.

Huerta, M.F., Harting, J.K., 1982. Tectal control of spinal cord activity: neuroanatomical demonstration of pathways connecting the superior colliculus with the cervical spinal cord grey. Prog. Brain Res. 57, 293-328.

Idrizbegovic, E., Bogdanovic, N., Canlon, B., 1999. Sound stimulation increases calcium-binding protein immunoreactivity in the inferior colliculus in mice. Neurosci. Lett. 259, 49-52.

Jones, B.N., Paabo, S., Stein, S., 1981. Amino acid analysis and enzymatic sequence determination of peptides by an improved o-phtalaldehyde precolumn labelling procedure. J. Liq. Chromatogr. 4, 556-586.

Kamphuis, W., Huisman, E., Wadman, W.J., Heizmann, C.W., Lopes da Silva, F.H., 1989. Kindling induced changes in parvalbumin immunoreactivity in rat hippocampus and its relation to long-term decrease in GABA-immunoreactivity. Brain Res. 479, 23-34.

Kanaseki, T., Sprague, J.M., 1974. Anatomical organization of pretectal nuclei and tectal laminae in the cat. J. Comp. Neurol. 158, 319-337.

Kawaguchi, Y., Katsumaru, H., Kosaka, T., Heizmann, C.W., Hama, K., 1987. Fast-spiking cells in rat hippocampus (CA1-region) contain the calcium-binding protein parvalbumin. Brain Res. 416, 369-374.

Kesner, R.P., 1966. Subcortical mechanisms of audiogenic seizures. Exp. Neurol. 15, 192-205.

Lasley, S.M., 1991. Roles of neurotransmitter amino acids in seizure severity and experience in the genetically epilepsy-prone rat. Brain Res. 560, 63-70.

Lawson, J.A., Vogrin, S., Bleasel, A.F., Cook, M.J., Bye, A.M., 2000. Cerebral and cerebellar volume reduction in children with intractable epilepsy. Epilepsia 41, 1456-1462.

Le Gal La Salle, G., Naquet, R., 1990. Audiogenic seizures evoked in DBA/2 mice induce c-fos oncogene expression into subcortical auditory nuclei. Brain Res. 518, 308-312.

Macías Fernandez, J.A., Gil-Verona, J.A., Franco Martín, M., 1992. Estudio neurofisiológico de crisis audiogenas en una cepa endogamica de hamsters dorados con epilepsia audiogena. Rev. Neurofisiol. Clin. 5, 13-18.

Martignoni, G., Pea, M., Brunelli, M., Chilosi, M., Zamo, A., Bertaso, M., Cossu-Rocca, P., Eble, J.N., Mikuz, G., Puppa, G., Badoual, C., Ficarra, V., Novella, G., Bonetti, F., 2004. CD 10 is expressed in a subset of chromophobe renal cell carcinomas. Mod. Pathol. 17, 1455-1463.

McAlpine, D., Martin, R.L., Mossop, J.E., Moore, D.R., 1997. Response properties of neurons in the inferior colliculus of the monaurally deafened ferret to acoustic stimulation of the intact ear. J. Neurophysiol. 78, 767-779. 
McCown, T.J., Greenwood, R.S., Frye, G.D., Breese, G.R., 1984. Electrically elicited seizures from the inferior colliculus: a potential site for the genesis of epilepsy. Exp. Neurol. 86, 527-542.

McHaffie, J.G., Anstrom, K.K., Gabriele, M.L., Stein, B.E., 2001. Distribution of the calcium-binding proteins calbindin D-28K and parvalbumin in the superior colliculus of adult and neonatal cat and rhesus monkey. Exp. Brain Res. 141, 460470.

Meredith, M.A., Miller, L.K., Ramoa, A.S., Clemo, H.R., Behan, M., 2001. Organization of the neurons of origin of the descending pathways from the ferret superior colliculus. Neurosci. Res. 40, 301-313.

Meredith, M.A., Stein, B.E., 1986. Visual, auditory, and somatosensory convergence on cells in superior colliculus results in multisensory integration. J. Neurophysiol. 56, 640-662.

Millan, M.H., Meldrum, B.S., Faingold, C.L., 1986. Induction of audiogenic seizure susceptibility by focal infusion of excitant amino acid or bicuculline into the inferior colliculus of normal rats. Exp. Neurol. 91, 634-639.

Mize, R.R., 1985. The analysis of immunohistochemical data. In: Mize, R.R. (Ed.), The Microcomputer in Cell and Neurobiology Research. Elsevier Science Publishing, Inc., New York, pp. 333-372.

Moschovakis, A.K., Karabelas, A.B., 1985. Observations on the somatodendritic morphology and axonal trajectory of intracellularly HRP-labeled efferent neurons located in the deeper layers of the superior colliculus of the cat. J. Comp. Neurol. 239, 276-308.

Mossop, J.E., Wilson, M.J., Caspary, D.M., Moore, D.R., 2000. Downregulation of inhibition following unilateral deafening. Hear. Res. 147, 183-187.

Palayoor, S.T., Seyfried, T.N., 1984. Genetic association between $\mathrm{Ca}^{2+}$-ATPase activity and audiogenic seizures in mice. J. Neurochem. 42, 1771-1774.

Park, W.M., Kim, M.J., Jeon, C.J., 2004. Ionotropic glutamate receptor GluR2/3 immunoreactive neurons in the cat, rabbit, and hamster superficial superior colliculus. Neurosci. Res. 49, 139-155.

Penny, J.E., Brown, R.D., Hodges, K.B., Kupetz, S.A., Glenn, D.W., Jobbe, P.C., 1983. Cochlear morphology of the audiogenicseizure susceptibility (AGS) or genetically epilepsy prone rat (GEPR). Acta Otolaryngol. 95, 1-12.

Penny, J.E., Brown, R.D., Wallace, M.S., Henley, C.M., 1986. Auditory aspects of seizure in the genetically epilepsy prone rat. Life Sci. 39, 887-895.

Ribak, C.E., Byun, M.Y., Ruiz, G.T., Reiffenstein, R.J., 1988. Increased levels of amino acid neurotransmitters in the inferior colliculus of the genetically epilepsy-prone rat. Epilepsy Res. 2, 9-13.

Ribak, C.E., Khurana, V., Lien, N.T., 1994. The effect of midbrain collicular knife cuts on audiogenic seizure severity in the genetically epilepsy-prone rat. J. Hirnforsch. 35, 303-311.
Ribak, C.E., Manio, A.L., Navetta, M.S., Gall, C.M., 1997. In situ hybridization for c-fos mRNA reveals the involvement of the superior colliculus in the propagation of seizure activity in genetically epilepsy-prone rats. Epilepsy Res. 26, 397-406.

Roberts, R.C., Ribak, C.E., Oertel, W.H., 1985. Increased numbers of GABAergic neurons occur in the inferior colliculus of an audiogenic model of genetic epilepsy. Brain Res. 361, 324-338.

Roberts, R.C., Ribak, C.E., 1986. Anatomical changes of the GABAergic system in the inferior colliculus of the genetically epilepsy-prone rat. Life Sci. 39, 789-798.

Ross, K.C., Coleman, J.R., 2000. Developmental and genetic audiogenic seizure models: behavior and biological substrates. Neurosci. Biobehav. Rev. 24, 639-653.

Russ, J., 1990. Computer-Assisted Microscopy: The Measurement and Analysis of Images. Plenum Press, New York.

Salvi, R.J., Wang, J., Ding, D., 2000. Auditory plasticity and hyperactivity following cochlear damage. Hear. Res. 147, 261-274.

Shank, R.P., Aprison, M.H., 1971. Post mortem changes in the content and specific radioactivity of several amino acids in four areas of the rat brain. J. Neurobiol. 2, 145-151.

Soria Milla, M.A., Macias Fernández, J.A., Aguirre Berrocal, A., Gomez Bosque, P., Gomez Carretero, M.E., 1987. Epilepsia audiogenica en una cepa endogamica de hamster dorado. Rev. Esp. Epilepsia 1, 27-33.

Stein, B.E., Meredith, M.A., 1993. The Merging of the Senses. MIT Press, Cambridge, MA.

Tews, J.K., Carter, S.H., Roa, P.D., Stone, W.E., 1963. Free amino acids and related compounds in dog brain: post-mortem and anoxic changes, effects of ammonium chloride infusion, and levels during seizures induced by picrotoxin and by pentylenetetrazol. J. Neurochem. 10, 641-653.

Van Buskirk, R.L., 1983. Subcortical auditory and somatosensory afferents to hamster superior colliculus. Brain Res. Bull. 10, 583-587.

Vater, M., Braun, K., 1994. Parvalbumin, calbindin D-28K, and calretinin immunostaining in the ascending auditory pathway of horseshoe bats. J. Comp. Neurol. 341, 534-558.

Wada, J.A., Terao, A., White, B., Jung, E., 1970. Inferior colliculus lesion and audiogenic seizure susceptibility. Exp. Neurol. 28, 326-332.

Wallace, M.T., Meredith, M.A., Stein, B.E., 1993. Converging influences from visual, auditory, and somatosensory cortices onto output neurons of the superior colliculus. J. Neurophysiol. 69, $1797-1809$.

Willott, J.F., Lu, S.M., 1980. Midbrain pathways of audiogenic seizures in DBA/2 mice. Exp. Neurol. 70, 288-299.

Winsky, L., Jacobowitz, D.M., 1995. Effects of unilateral cochlea ablation on the distribution of calretinin mRNA and immunostaining in the guinea pig ventral cochlear nucleus. J. Comp. Neurol. 354, 564-582.

Yao, W., Godfrey, D.A., 1997. Densitometric evaluation of markers for cholinergic transmission in rat superior olivary complex. Neurosci. Lett. 229, 21-24. 